\title{
Gear micropitting: model and validation
}

\author{
J. A. Brandão ${ }^{1}$, J. H. O. Seabra ${ }^{2}$ \& M. J. D. Castro ${ }^{3}$ \\ ${ }^{1}$ INEGI-Instituto de Engenharia Mecânica e Gestão Industrial, \\ Porto, Portugal \\ ${ }^{2}$ FEUP_Faculdade de Engenharia da Universidade do Porto, \\ Departamento de Engenharia Mecânica e Gestão Industrial, \\ Porto, Portugal \\ ${ }^{3}$ ISEP_Instituto Superior de Engenharia do Porto, \\ Departamento de Engenharia Mecânica, Porto, Portugal
}

\begin{abstract}
A numerical model for the prediction of surface initiated damage on gear tooth flanks (micropitting and mild wear) is presented. This model hinges on a model of the mixed film lubrication regime and on the application of the Dang Van highcycle multi-axial fatigue criterion. The numerical model developed was applied to the prediction of micropitting and mass loss of gear teeth in an actual micropitting test.
\end{abstract}

Keywords: gears, surface fatigue, micropitting, numerical model, wear.

\section{Introduction}

Micropitting is best described as the appearance of minuscule cavities on the surface of a gear tooth, most often near and bellow the pitch line. They are thought to be caused by the propagation of micro-cracks originating from the tooth surface and swiftly reemerging so as to form a pit with a typical depth of $10 \mu \mathrm{m}[1,2]$. Widespread micropitting severely increases the noise level of a gear box, and in some instances may sufficiently weaken the tooth subsurface for a whole layer to be removed at once, introducing a shape defect.

Because of the small size of the pits, the crack propagation time is correspondingly small and the fatigue life is dominated by the initiation time. Additionally, the fact that the phenomenon remains circumscribed within the first few tens of micrometres below the tooth surface renders it extremely sensitive to the contact 
stress perturbations caused by the roughness of the surfaces and forces one to consider a lubrication model able to deal with such perturbations: a mixed film lubrication model.

For all these reasons, a numerical model was introduced that attempts to predict the onset of micropitting fatigue cracks on the surface of spur gear teeth by taking into account the geometry, roughness, residual stresses induced by manufacturing processes and the lubrication regime and by applying a fatigue initiation criterion, the Dang-van multi-axial fatigue initiation criterion.

This work is a revisitation of that previously presented in [3] and [4], where the model, with its underlying principles and methodology were put forward in great detail. The reader is invited to consult these papers if he finds the somewhat more sketchy presentation offered here insufficient. This revisitation is rendered opportune because in the time since the presentation of these works some new information came to light in [5] regarding the lubrication of the mineral oil used as an example in these works.

\section{Numerical model}

The overall workings of the models are shown schematically in figure 1. As can be observed, the model is comprised of two great blocks: one for the calculation of the elastic stresses at each instant of the meshing of the gears and another for the application of the Dang-Van high cycle fatigue initiation model to each point within the depth of $30 \mu \mathrm{m}$ inside the tooth surface. It is not within the scope of this document to explain the model in detail, which is fully exposed in [4]. Instead, the readers attention is directed to several elements that must be provided if the model is to have adequate accuracy:

- the exact surface geometry, including its roughness;

- the manufacturing induced residual stresses;

- the boundary friction coefficient;

- a load sharing function that balances the load between the full film elastohydrodynamic (EHD) and the boundary lubrication cases;

- the rheological properties of the lubricant from which the full film EHD friction coefficient may be derived.

The first through third item are resolved through measurement. In particular, the third item is pointed to the reader's attention because it was the source of some uncertainty in [4] that [5] has dispelled. As for the remaining items, as well as the actual fatigue initiation criterion, they will be briefly discussed in what follows for the convenience of the reader.

\subsection{Lubrication model}

\subsubsection{Contact pressure distribution}

While in contact, a pair of meshing gear teeth is separated by a film of lubricant (most often, and the only case considered here, a lubricating oil). The film may separate completely the teeth surfaces, in which case the lubrication is full film 


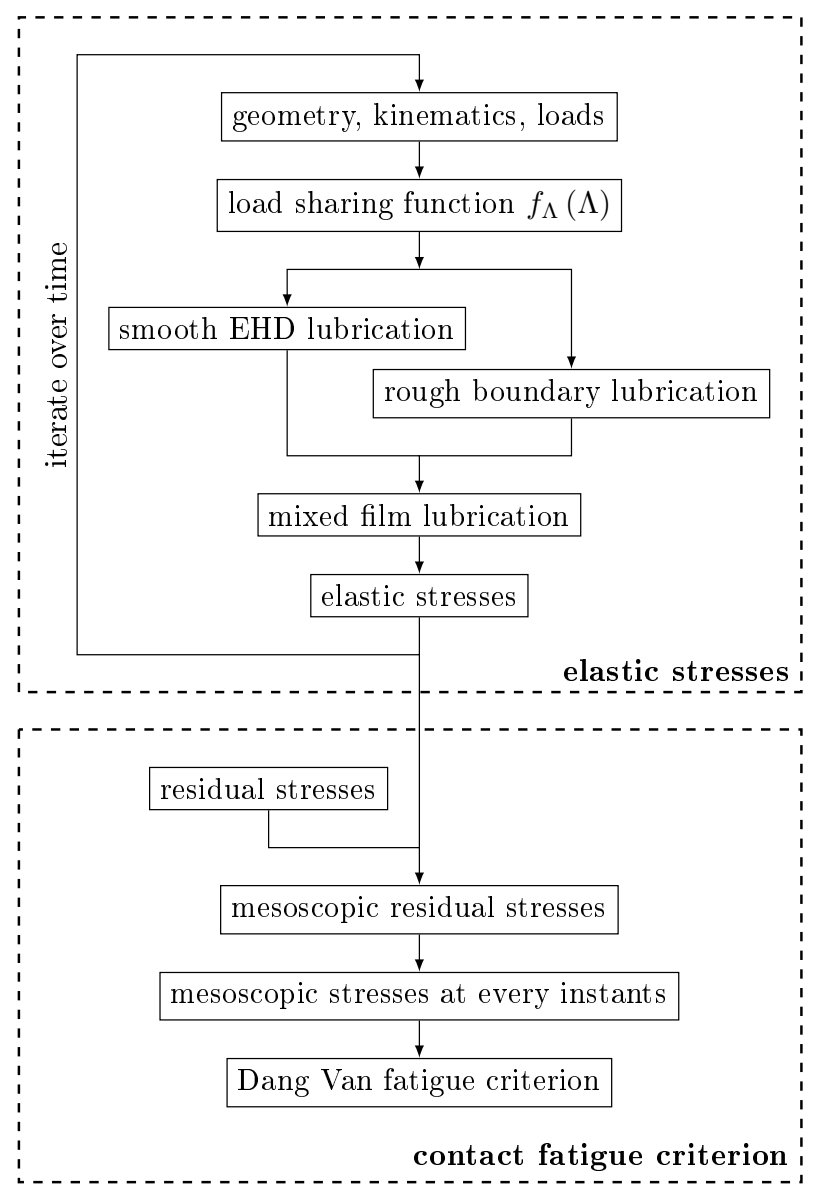

Figure 1: Diagram of the numerical model.

EHD (elasto-hydrodynamic); it may cease to bear load and only contribute to a decrease in the friction coefficient between the surfaces, in which case we have boundary lubrication; and it may be somewhere between those extremes, in which case we have mixed film lubrication, in which, while some separation of the surfaces is provided by the oil, direct contact still occurs between roughness features of the surfaces. It is generally acknowledged that the contact lubrication regime is controlled by the specific lubricant film thickness $(\Lambda)$, calculated by:

$$
\Lambda=\frac{\text { theoretical thickness of the film in EHD lubrication }}{\text { composite RMS roughness of the surfaces }}
$$

When $\Lambda$ is high $(\Lambda>3)$, the lubrication is full film elasto-hydrodynamic, when it is low $(\Lambda<0.1)$, we have boundary lubrication. Intermediate values define the range of mixed film lubrication. 
The mixed film lubrication model proposed in [3] consists of a radical simplification whereby, instead of solving the full problem, a functions $f_{\Lambda}$ of $\Lambda$ is posited so that:

$$
p=f_{\Lambda}(\Lambda) p^{\text {EHD.T }}+\left[1-f_{\Lambda}(\Lambda)\right] p^{\text {BDR.T }}
$$

in which, $p^{\text {EHD.T }}$ is the theoretical contact pressure in full film EHD when ignoring the surface roughness, $p^{\text {BDR.T }}$ the theoretical pressure in boundary lubrication when ignoring any bearing film and $p$ the actual contact pressure.

\subsubsection{Contact shear stress distribution}

The shear stress within the contact is obtained as a sum of the contribution of the full film portion and the boundary portion.

It is generally accepted that in boundary lubrication the friction coefficient $\mu^{\mathrm{BDR}}$ is essentially insensitive to variations of temperature, rolling velocity and slide-toroll ratios and its value is to be found in a range approximately between 0.08 and 0.15 . The actual values must be determined in each case of combination of lubricant and type of surface roughness with the aid of traction tests (see [5]).

All this means that the portion of the shear strain contributed by the boundary lubrication is:

$$
\tau^{\mathrm{BDR}}=\mu^{\mathrm{BDR}}\left(1-f_{\Lambda}\right) p^{\mathrm{BDR} . \mathrm{T}}
$$

In the case of the full film EHD contribution to the shear stress, things are more complex. Within a lubricant, its rheology establishes the relation of the shear stress with several other variables. In the present case the type of rheological law used is a Bair - Winer visco-plastic law:

$$
\dot{\gamma}=\frac{\tau}{\eta} \frac{-\ln \left(1-\left|\tau / \tau_{L}\right|\right)}{\left|\tau / \tau_{L}\right|}
$$

where $\tau$ is the shear stress, $\dot{\gamma}$ the shear strain rate, $\eta$ is the low shear dynamic viscosity and $\tau_{L}$, the limiting shear stress.

The low shear dynamic viscosity is here supposed to follow the Roelands viscosity law:

$$
\ln \frac{\eta}{\eta_{0}}=\left(\ln \eta_{0}+9.67\right)\left\{\left(\frac{T-138}{T_{0}-138}\right)^{-S_{0}}\left(1+\frac{p}{196}\right)^{Z}-1\right\}
$$

where $Z$ and $S_{0}$ are non-dimensional parameters of the oil, $T_{0}$ is a reference temperature in $\mathrm{K}, \eta_{0}$ is the dynamic viscosity in $\mathrm{Pa} \cdot \mathrm{S}$ at the reference temperature an atmospheric pressure, $T$ is the temperature of the lubricant in $\mathrm{K}, p$ is the pressure in $\mathrm{GPa}$ and $\eta$ is the dynamic viscosity in $\mathrm{Pa} \cdot \mathrm{s}$.

The limiting shear stress is supposed to follow a Barus type law:

$$
\tau_{L}=\tau_{L 0} \exp \left(\alpha_{\tau} p+\beta_{\tau}\left(T^{-1}-T_{0}^{-1}\right)\right)
$$

where $\tau_{L 0}$ is the limiting shear stress at atmospheric pressure and reference temperature $T_{0}, \alpha_{\tau}$ and $\beta_{\tau}$ parameters to be determined empirically that account for the influence of pressure and temperature. 
The pressure $p$ is already known and equal to $\left(f_{\Lambda} \cdot p^{\text {EHD.T }}\right)$. The strain rate is derived from the thickness of the theoretical full film EHD lubrication. The temperature is obtained by solving the thermal equation:

$$
\rho C_{p} u \frac{\partial T}{\partial x}=k \frac{\partial^{2} T}{\partial z^{2}}+\tau \cdot \dot{\gamma}
$$

where $\rho$ is the volumic mass, $C_{p}$ the heat capacity, $u$ the mean velocity and $k$ the thermal conductivity.

The thermal equation and the rheological equation are coupled and, after having been simultaneously solved, yield the shear stress $\tau^{\mathrm{EHD}}$ and the temperature $T$ within the contact.

Finally, the actual shear stress is:

$$
\tau=\tau^{\mathrm{EHD}}+\tau^{\mathrm{BDR}}
$$

\subsection{Dang-Van criterion}

The Dang Van fatigue criterion [6] makes use of the mesoscopic stresses(stresses observed at the scale of the material grain) to predict the onset of high-cycle fatigue in a material point. These mesoscopic stresses are derived from the macroscopic ones (those obtained from ordinary continuum mechanics) as explained in $[6,3]$. Knowing these mesoscopic stresses, the verification of initiation at any material point is expressed by the equation:

$$
\tau_{\max }+\alpha_{\mathrm{DV}} p_{H}>\beta_{\mathrm{DV}}
$$

where $\tau_{\max }$ is the maximum mesoscopic shear stress and $p_{H}$ the hydrostatic stress (the negative of the hydrostatic pressure). The parameters $\alpha_{\mathrm{DV}}$ and $\beta_{\mathrm{DV}}$ in equation (9) are fatigue properties of the material to be determined by performing a reversed torsion and alternating bending tests.

Whenever this expression is true, an initiation point is found. This is illustrated in figure 2, where the position of the mesoscopic stress state of a material point during a hypothetical load cycle on the $p_{H} / \tau_{\max }$ plane is shown. It is seen that the path of the stress crosses the straight line delimiting the safety zone.

\section{Gear micropitting: test and simulation}

The numerical model's results are compared in this section to a gear micropitting test performed by Cardoso on an FZG test rig [7, 8]. The FZG type C gears used in the test were of DIN 20MnCr5 carburizing steel. The carburizing treatment left them with a $0.8 \mathrm{~mm}$ thick carburized layer and a surface hardness of 60 HRC. The gear oil is an ISO VG 150 paraffinic mineral oil that contains additives to increase its resistance to micropitting. Some of its properties are reproduced from [7, Table 1.1] in Table 1.

Table 2 lists the operating conditions for each load cycle. In addition the test procedure as well as the simulation procedure are described in Table 3. 
Table 1: Gear oil properties.

\begin{tabular}{lcccc}
\hline Parameter & Method & Desig. & Value \\
\hline Base oil & DIN 51451 & & Paraffinic mineral oil \\
$\begin{array}{l}\text { Density at } 15{ }^{\circ} \mathrm{C} \\
\text { Kinematic Viscosity }\end{array}$ & at & DIN 51757 & $\rho_{15}$ & $0.894 \mathrm{~g} \mathrm{~cm}^{-3}$ \\
$40{ }^{\circ} \mathrm{C}$ & & & $\nu_{40}$ & $153.6 \mathrm{cSt}$ \\
$\begin{array}{l}\text { Kinematic Viscosity } \\
100{ }^{\circ} \mathrm{C}\end{array}$ & at & DIN 51562 & $\nu_{100}$ & $14.4 \mathrm{cSt}$ \\
Viscosity Index & & DIN ISO 2909 & VI & 96 \\
\hline
\end{tabular}

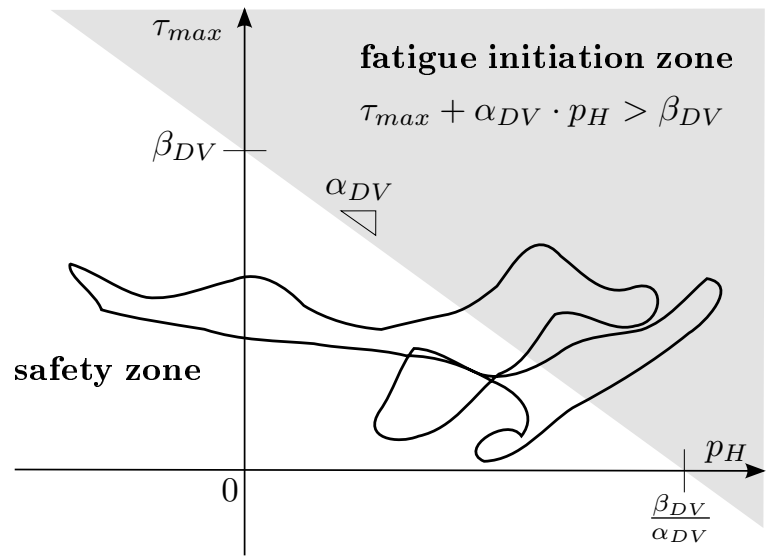

Figure 2: Position of the mesoscopic stress state of a material point during a load cycle on the $p_{H} / \tau_{\max }$ plane. The shaded half-plane represents the area where the Dang Van criterion is violated. Thus, the parts of the cycle placed in the shaded area violate the criterion.

Figure 3 shows the approximate positions where the tooth flank surface roughness measurements were made: on the left, those performed before load stage $\mathrm{K} 3$ and on the right those performed between successive load cycles (K6-K8 and K8-K9).

In addition to the parameters already presented, several others must be determined. In particular, the oil properties to be used in equations (5) and (6) must be known, as well as the boundary friction coefficient and the load sharing function.

The boundary friction coefficient of the oil was determined in [5] and was found to be approximately 0.09 . The remaining parameters were obtained through an indirect method described in [4]. 
Table 2: Operating conditions of the micropitting test.

\begin{tabular}{|c|c|c|c|c|}
\hline load stage & K3 & K6 & K8 & K9 \\
\hline normal load $F_{N}$ & $851 \mathrm{~N}$ & $2924 \mathrm{~N}$ & $5073 \mathrm{~N}$ & $6373 \mathrm{~N}$ \\
\hline oil temperature & $80^{\circ} \mathrm{C}$ & & $90{ }^{\circ} \mathrm{C}$ & \\
\hline duration & $1 \mathrm{~h}$ & & $16 \mathrm{~h}$ & \\
\hline $\begin{array}{l}\text { number of cycles undergone by } \\
\text { the driving gear }\end{array}$ & $135 \cdot 10^{3}$ & & $2.16 \cdot 10^{6}$ & \\
\hline $\begin{array}{l}\text { angular velocity of the driving } \\
\text { gear } n_{1}\end{array}$ & \multicolumn{4}{|c|}{2250 RPM } \\
\hline $\begin{array}{l}\text { angular velocity of the driven } \\
\text { gear } n_{2}\end{array}$ & \multicolumn{4}{|c|}{1500 RPM } \\
\hline
\end{tabular}

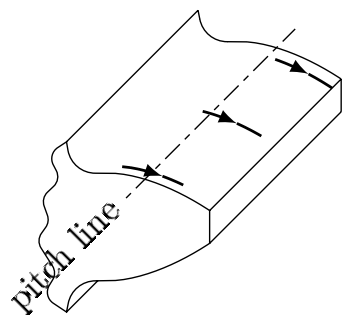

(a)

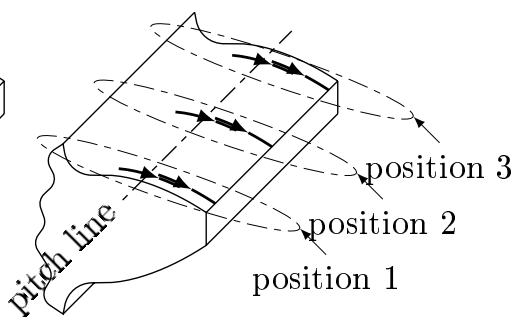

(b)

Figure 3: Positions of roughness measurement. a) the roughness measurements before stage K3. b) roughness measurements between load stages: each position includes one measurement below the pitch line and one above it.

For the application of the Dang Van criterion, its fatigue parameters $\alpha_{\mathrm{DV}}$ and $\beta_{\mathrm{DV}}$ must be known as well. Of those, only the value of $\alpha_{\mathrm{DV}}(0.987)$ was available in the open literature. Because of this each load stage was repeatedly simulated with different values of $\beta_{\mathrm{DV}}$ so as to scan the range $400 \mathrm{MPa}<\beta_{\mathrm{DV}}<900 \mathrm{MPa}$.

\section{Comparison of the simulation with the gear test results}

The test afforded two types of quantitative results: mass loss of the gears and roughness profiles at the end of each load stage. Neither is directly comparable to the types of results of the Dang-Van criterion which merely identifies loci of fatigue crack initiation.

A tentative solution was adopted here and will now be described. Consider figure 4 , which shows the mechanism of crack propagation of a micropit proposed 
Table 3: Gear micropitting test procedure and corresponding simulation procedure.

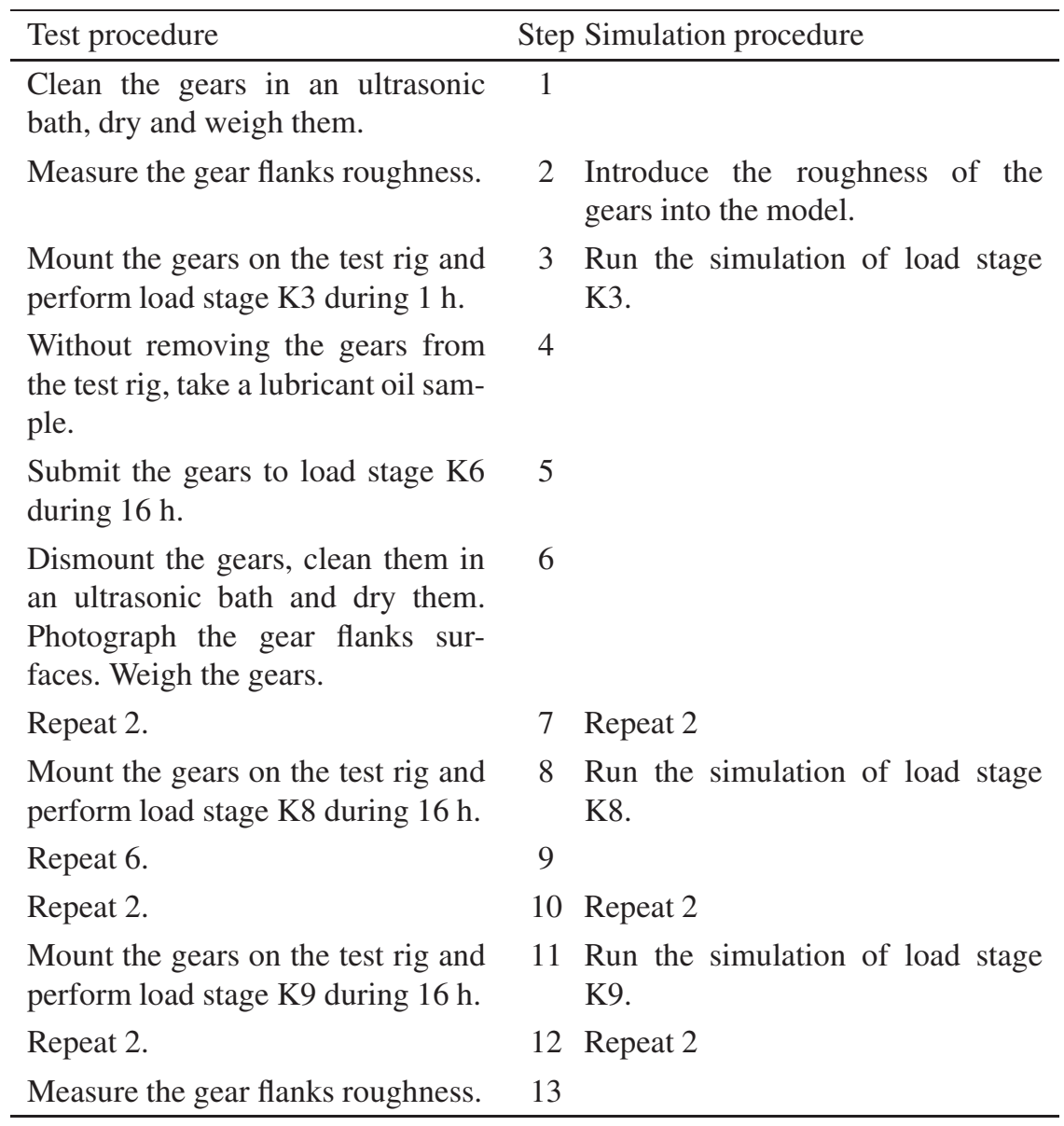

in [1] in juxtaposition to an area of a tooth subsurface where a continuous patch of fatigue criterion violation is predicted. Both the dimensions and shapes in the sub-figures are similar. From here to assuming that they are essentially the same is a short step that the authors gladly take. Thus, in all that follows, whenever an area of fatigue initiation is found, it is assumed that the corresponding material will have been removed by the end of the load stage.

Table 5 shows the comparison of the measured mass loss in the driving gear at the end of each load stage with that predicted applying this propagation model considering two possible values of $\beta_{\mathrm{DV}}$.

Even though there are no actual measurements against which to compare it, the value from the simulation of load stage $\mathrm{K} 3$ by itself is plausible, since it falls 
Table 4: Parameters of the simulation.

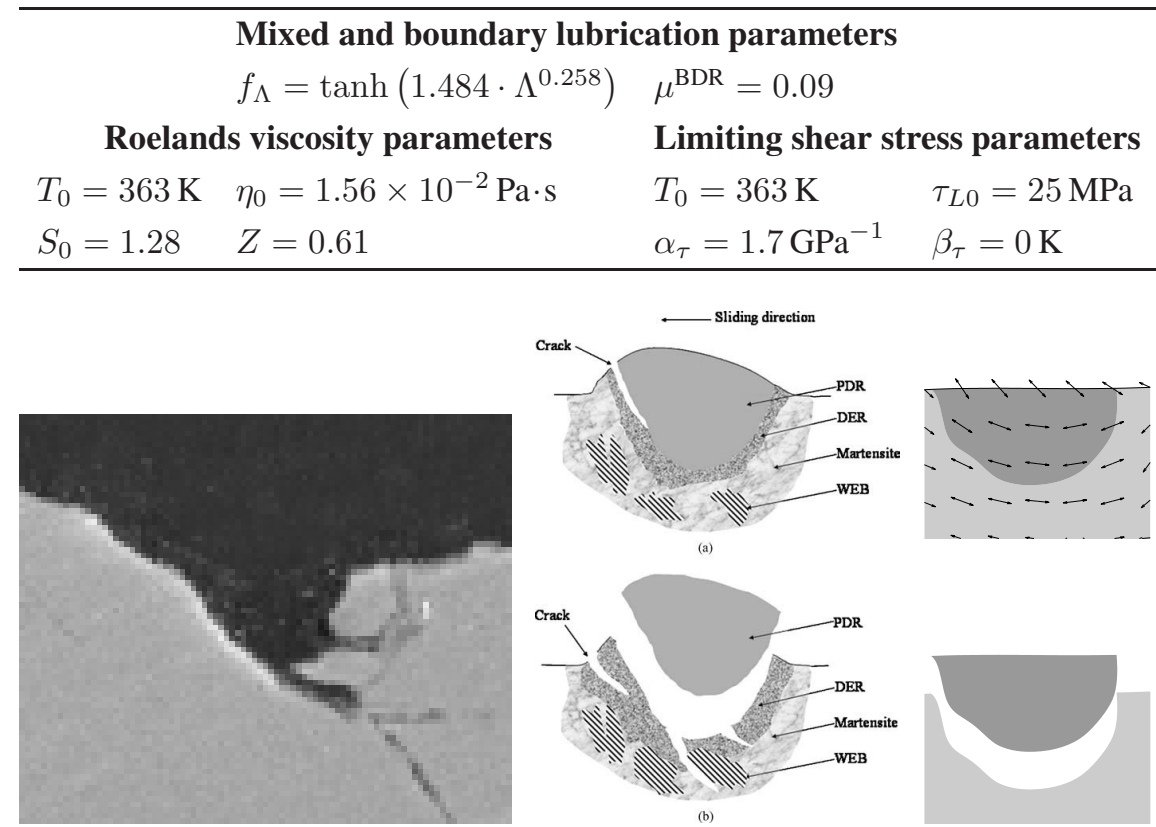

Figure 4: Approximate mechanism of crack propagation. (left) Photograph of the section of a micropit. (centre) Crack propagation mechanism according to Oila and Bull [1]. The crack propagates along the boundary between the plastic deformation region (PDR) and the dark etching region (DER). These, as well as the white etching band (WEB) are phases due to the change in steel micro-structure. (right) Approximate crack propagation mechanism adopted here: the crack propagates on the boundary of a Dang Van fatigue initiation patch until the patch is removed from the tooth.

well below the value measured at the end of the consecutive run of load stages $\mathrm{K} 3$ and K6.

The most dramatic fact to emerge from the data can be better observed with the help of figure 5, where the values of gear mass loss for the whole gear are also represented: the prediction of mass loss when $\beta_{\mathrm{DV}}=400$ is nearly flawless. Not only that, but for other values of $\beta_{\mathrm{DV}}$, the predicted mass loss curves stay resolutely parallel to the curve connecting the measured values. This advocates strongly for a very direct correlation of the Dang Van fatigue initiation area with the mass loss due to micropitting and, indeed, to a certain extent mild wear.

In figure 6 are shown as gray boxes the range of measured values of the roughness parameter $R_{v k}$ below the pitch line of one tooth. Shown as well are 
34 Tribology and Design

Table 5: Comparison of the measured mass loss at the end of each load stage with the predicted one.

\begin{tabular}{lcccc}
\hline & $\begin{array}{c}\mathrm{K} 3 \\
{[\mathrm{mg}]}\end{array}$ & $\begin{array}{c}\mathrm{K} 3+\mathrm{K} 6 \\
{[\mathrm{mg}]}\end{array}$ & $\begin{array}{c}\mathrm{K} 8 \\
{[\mathrm{mg}]}\end{array}$ & $\begin{array}{c}\mathrm{K} 9 \\
{[\mathrm{mg}]}\end{array}$ \\
\hline \multicolumn{2}{l}{ model prediction with } & & & \\
$\beta_{\mathrm{DV}}=500 \mathrm{MPa}$ & 0.60 & $\mathrm{~N} / \mathrm{A}$ & 2.756 & 2.059 \\
$\beta_{\mathrm{DV}}=400 \mathrm{MPa}$ & 1.41 & $\mathrm{~N} / \mathrm{A}$ & 6.230 & 5.162 \\
test measurements & N/A & $3 \pm 0.5$ & $6 \pm 0.5$ & $5 \pm 0.5$ \\
\hline
\end{tabular}

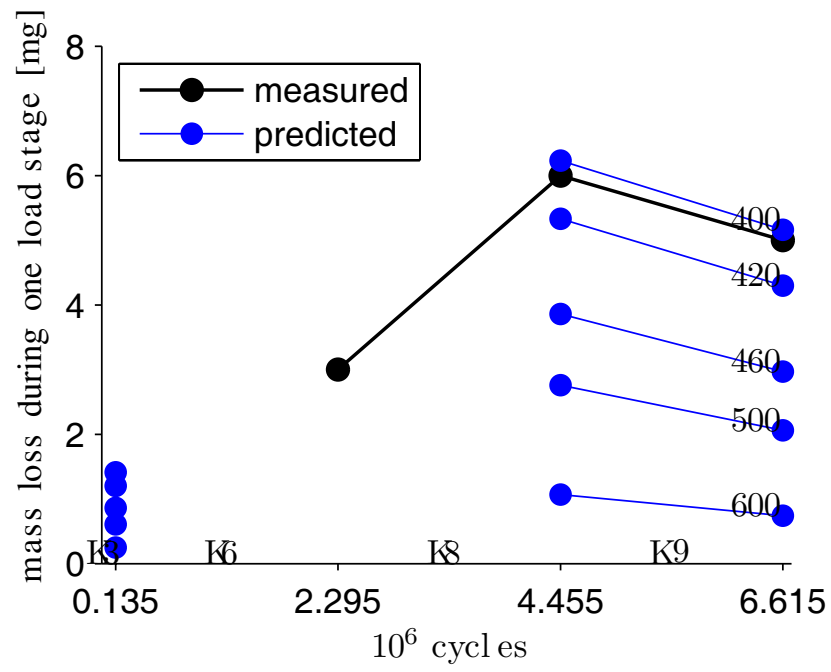

Figure 5: Mass loss of the driving gear in each stage: measured values and predicted ones for several simulation cases in which $\mu^{\mathrm{BDR}}=0.09$ and $\beta_{\mathrm{DV}}$ varies from 400 to $600 \mathrm{MPa}$.

the evolution of that same parameter as predicted by the proposed propagation model applied to several possible values of $\beta_{\mathrm{DV}}$. Acceptable values of roughness parameters should fall within the range of measured roughness shown as shaded boxes. This is the case for the curves that match $\beta_{\mathrm{DV}}>500$. Unfortunately, those values also produce poor approximations to the mass loss. Once more "virtue is in the middle": there must be a value somewhere between 400 and $600 \mathrm{MPa}$ that corresponds to the true $\beta_{\mathrm{DV}}$.

However, some reflections with bearing on this quandary should be put forward. While the impression was given that the roughness measurement positions shown in figure 3 were precisely the same from load stage to load stage, this is hardly the 


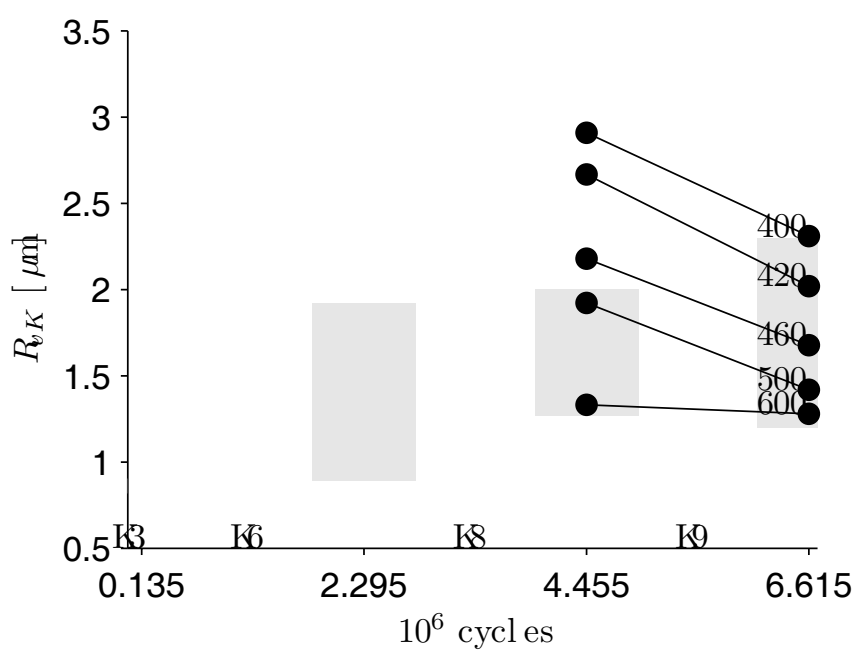

Figure 6: A tooth's evolution of roughness parameter $R_{v k}$ below the pitch line. The shaded box show the range of the multiple measured values.

case: the operator performing the positioning of the gear at the beginning of each roughness measurement cannot be expected to accomplish this within a precision of a fraction of a millimetre. In practice, this means that an attempt is being made to uses one profile at a certain position as a starting point to predict another profile in a different position. On the other hand, this fact has much less bearing on the calculation of the mass loss, a global, integrated value much less sensitive to such slight variations.

\section{Conclusion}

A micropitting prediction model was applied in the simulation of an actual gear micropitting test. It has shown that an interval of values of $\beta_{\mathrm{DV}}$ can be identified within which the model is able to account for both the mass loss and, with some reservations, the roughness alterations on the surface of a gear. Thus, the carburized layer's fatigue property most needed for the application of the model is bracketed by values of approximately $400 \mathrm{MPa}$ and $600 \mathrm{MPa}$. This goes a long way to confirm the validity of the model put forward here.

\section{Acknowledgements}

The authors wish to thank the "Fundação para a Ciência e Tecnologia" from the Portuguese Administration for its financial support to this work through the research contract "PTDC/EME-PME/66185/2006". 


\section{References}

[1] Oila, A. \& Bull, S., Phase transformations associated with micropitting in rolling/sliding contacts. Journal of Materials Science, 40(18), pp. 47674774(8), September 2005.

[2] Oila, A. \& Bull, S.J., Assessment of the factors influencing micropitting in rolling/sliding contacts. Wear, 258(10), pp. 1510-1524, 2005.

[3] Brandão, J.A., Seabra, J.H.O. \& Castro, J., Surface initiated tooth flank damage. part I: Numerical model. Wear, 268(1-2), pp. 1-12, 2010.

[4] Brandão, J.A., Seabra, J.H.O. \& Castro, J., Surface initiated tooth flank damage. part II: Prediction of micropitting initiation and mass loss. Wear, 268(1-2), pp. 13-22, 2010.

[5] Brandão, J.A., Meheux, M., Ville, F., Seabra, J.H.O. \& Castro, J., Experimental traction and stribeck curves of mineral, pao and ester based fully formulated gear oils. IRF2009 - 3rd International Conference on Integrity, Reliability \& Failure, Porto, 2009.

[6] Dang Van, K., Griveau, B. \& Message, O., On a new multiaxial fatigue limit criterion: theory and application. Biaxial and Multiaxial Fatigue, eds. M. Brown \& K. Miller, EGF 3, Mechanical Engineering Publications, London, pp. 479-498, 1989.

[7] Cardoso, N., Mechanical performance of biodegradable, low toxicity ester based industrial oils. Master's thesis, Faculdade de Engenharia da Universidade do Porto, 2007.

[8] Martins, R. \& Seabra, J., Gear micropitting performance of mineral and biodegradable ester gear oils. Industrial Lubrication and Tribology, 60(6), pp. 286-292, 2008. 